\title{
Um panorama do elemento Tecnologia na Educação CTS e o ensino de Ciências
}

\author{
An Overview of the Technology Element in STS Education and Science \\ Teaching
}

\section{Resumo}

O campo de estudos Ciência, Tecnologia e Sociedade (CTS) vem sinalizando, nos últimos anos, obstáculos para implementar o acrônimo CTS como um todo no ensino de Ciências, principalmente o segundo elemento, Tecnologia (T). A fim de contribuir com a discussão acerca das relações que envolvem essa tríade, realizamos uma ampla revisão bibliográfica em bases de dados e periódicos da área de ensino, buscando produções que abordaram a Tecnologia na Educação CTS e o ensino de Ciências entre os anos de 2010-2020. Para tal, foi necessário analisar qual sentido de tecnologia prevalece nos resultados dessas produções. Foram selecionados 39 artigos, considerando os critérios de inclusão. Para o tratamento analítico desse corpus, utilizamos elementos da análise de conteúdo de Bardin (1977). As categorias emergentes a posteriori foram: Natureza do Trabalho; Concepção de Tecnologia; e Tecnologia na Abordagem CTS. A maioria dos trabalhos analisados traz concepções restritas/simplistas de tecnologia em seus resultados, seja de professores em exercício, professores em formação ou estudantes da educação básica. Assim, inferimos a necessidade de uma maior difusão e ascensão do sentido atribuído à tecnologia, considerando sua amplitude de maneira crítica. Abordagens que partem desse entendimento no Ensino de Ciências estabelecem de fato uma efetiva educação científicotecnológica.

Palavras chave: Educação CTS; Tecnologia crítica; Natureza da Tecnologia.

\section{Abstract}

The Science, Technology and Society (STS) field of studies has been showing, in recent years, obstacles to implementing the acronym STS as whole in the teaching in Science, especially the second element: Technology $(\mathrm{T})$. In order to contribute to the discussion about the relationships that involve this triad, we carried out a broad bibliographic review in databases and periodicals in the field of education, seeking Productions that addressed Technology in STS Education and Science teaching between the years of 2010 - 2020. For that, it was necessary to analyze which sense of technology prevails in the results of these Productions, 39 articles were selected, considering the inclusion criteria. For the analytical treatment of this corpus, we used elements from the content analysis of Bardin (1977). The categories that

\footnotetext{
${ }^{1}$ Universidade Federal do Rio Grande do Norte | dioginyscesar@gmail.com

${ }^{2}$ Universidade Federal do Rio Grande do Norte | josivaniamd@yahoo.com.br
} 
emerged posteriori were: Nature of Work; Technology Conception; and Technology in the STS Approach. Most of the analyzed works bring restricted/simplistic conceptions of technology in their results, whether from practicing teachers, teachers training or students of basic education. This, we infer the need for a greater diffusion and rise of the meaning attributed to technology, considering its amplitude in a critical way. Approaches that start from this understanding in Science Teaching establish in fact an effective scientifictechnological education.

Keywords: STS Education; Critical Technology; Nature of Technology.

\section{Introdução}

A ciência e tecnologia influenciam diretamente em nosso modo de vida. O desenvolvimento científico e tecnológico trouxe muitos benefícios à humanidade, contudo, caso olhemos sobre outra ótica, podemos notar que estes trouxeram consigo diversas consequências sociais, ambientais e econômicas, pois muitas vezes na história da humanidade não foram utilizados de forma benéfica, ora conscientes ou não.

Dentre as consequências, apontamos alguns problemas socioambientais, como o excesso de lixo inorgânico, espacial e eletrônico, exploração exacerbada dos recursos naturais sem o seu uso sustentável, contaminações de mares e rios, acidentes nucleares, emissões de gases, agrotóxicos poluentes derivados do avanço desenfreado das indústrias e agronegócio, dentre outros, que acabam substancialmente alterando as relações existentes entre os seres vivos e o meio ambiente.

É a partir do contexto apresentado que destacamos que os cursos de formação de professores da área das Ciências da Natureza e a Educação Básica devem abordar e refletir criticamente acerca do desenvolvimento da ciência e tecnologia, e também ter como apoio as diferentes dimensões e concepções epistemológicas da natureza da ciência e tecnologia. Isso com o intuito de mediar saberes que possam auxiliar a formação da alfabetização científica e tecnológica dos alunos, para que assim esses sujeitos possam se tornar autônomos, críticos e desenvolver melhor suas tomadas de decisões na sociedade, frente a esse desenvolvimento no futuro (RODRÍGUEZ; DEL PINO, 2017).

Nessa direção, a literatura da área de ensino de Ciências Naturais sugere diferentes alternativas metodológicas para enfrentar a complexidade das questões planetárias. Dentre essas tendências metodológicas, a Educação Ciência, Tecnologia e Sociedade (CTS) é um caminho que possibilita aos sujeitos um olhar e interpretação abrangente para compreender as relações existentes dos aspectos sociais da ciência e tecnologia, bem como as consequências socioambientais trazidas por essas relações (BAZZO; LINSINGEN; PEREIRA, 2003).

Por outro lado, apesar de essa abordagem possibilitar a contextualização e a interdisciplinaridade entre diferentes áreas do conhecimento, alguns autores brasileiros que estudam a Educação CTS, como Galieta-Nascimento e Linsingen (2006); Cassiani et al. (2014); Geremias (2021); Abreu e Strieder (2016); Firme (2020); e Lorenzetti (2021), sinalizaram haver pouca atenção dada a um dos elementos que compõem a tríade CTS, o segundo elemento: $(T)$ - tecnologia, que, por vezes, é tratada em um plano de fundo em tal abordagem.

Essa ocorrência se dá porque algumas propostas de Educação CTS centram suas discussões e enfatizam apenas a relação entre (C e S) - Ciência e Sociedade. Dessa maneira, 
a visualização do seu segundo elemento, $(T)$ - tecnologia, fica silenciada e vista como uma aplicação da ciência (BOCHECO, 2011; CASSIANI et al., 2014).

Em virtude dessa constatação, este estudo buscou realizar uma ampla revisão da literatura, entre o decênio 2010 a 2020, em bases de dados e periódicos da área de ensino, com o objetivo de evidenciar publicações que abordaram a tecnologia na Educação CTS e o ensino de Ciências no Ensino Fundamental Anos Finais, Ensino Médio e Ensino Superior. Para tal, foi necessário analisar qual o sentido de tecnologia que prevalece nos resultados dessas produções. Nessa perspectiva, os tópicos que sucedem darão destaque ao elemento tecnologia de maneira crítica e ampla do qual nos apoiamos.

\section{Tecnologia como um conceito amplo}

A tecnologia, assim como os estudos CTS, é, segundo Acevedo (1998), um termo polissêmico, complexo e possui múltiplas interpretações. Seu uso cotidiano e atual é rotineiro, o que acaba confundindo seu conceito com termos como técnica e ciência, situação que dificulta seu real significado.

A tecnologia não é meramente uma aplicação da ciência, algo que seja neutro, ou simplesmente um artefato tecnológico, como corriqueiramente e intuitivamente relacionamos a ela. O conceito de tecnologia é amplo e o campo de estudos CTS deve considerar seu sentido ampliado. Para Bazzo (2014, p. 138), qualquer definição de tecnologia, antes de mais nada, deveria considerar que:

Tecnologia tem relação com a ciência, com a técnica e com a sociedade; Tecnologia integra elementos materiais - ferramentas, máquinas, equipamentos - e não materiais - saber fazer, conhecimentos, informações, organização, comunicação e relações interpessoais; Tecnologia tem relações com fatores econômicos, políticos e culturais; Evolução da tecnologia é inseparável das estruturas sociais e econômicas de uma determina sociedade.

Nesse sentindo, podemos observar que a tecnologia não se limita a apenas um equipamento sofisticado do nosso tempo, ela abarca essa concepção, mas não se limita apenas a isso. Esse equívoco é gerado por associarmos a tecnologia a um resultado ou a produtos industriais de natureza material, que se manifesta a partir dos objetos/artefatos tecnológicos que fazem parte do nosso dia a dia, como, por exemplo: telefones, computadores, máquinas e automóveis. Em todos esses exemplos, encontramos uma imagem simplista e convencional da tecnologia (BAZZO; LINSINGEN; PEREIRA, 2003).

No entanto, a tecnologia não se limita somente aos artefatos tecnológicos. Para o professor e filósofo Alberto Cupani (2020, p. 82), há quatro dimensões de tecnologia que se manifestam "Como artefatos e sistemas, como certo tipo de conhecimento, como atividades específicas e como determinada atitude humana perante a realidade natural ou social. Em conjunto, elas configuram nosso mundo, o mundo tecnológico".

É natural fazermos uma ligação intuitiva da tecnologia a artefatos. Entretanto, caso reflitamos melhor nessa dimensão, todos os objetos/materiais produzidos pelo homem são um tipo de tecnologia, desde a pedra que foi amolada para caçar durante a pré-história, até as nossas roupas nos dias atuais são um tipo de tecnologia que se constitui como manifestações tecnológicas construídas nas civilizações (CUPANI, 2016; MITCHAM,1994). 
A tecnologia, em uma outra dimensão, é vista por Cupani (2016) como um modo específico de conhecimento. Ela surgiu antes da ciência e tem como resultado final avanços de produtos, de patentes e de ferramentas. Ela se constitui, portanto, um campo específico e separado da ciência, embora saiba-se que, em muitos casos, para que seu avanço ocorra, são incorporados saberes científicos, ou, da mesma maneira, é necessário a utilização de saberes tecnológicos para que a ciência avance, e, nesse sentido, podemos dizer que estes possuem conhecimentos específicos e características que as distinguem, mas que estão intimamente interligados.

Além disso, as tecnologias fazem parte e molda a nossa cultura e a maneira que nos relacionamos com o mundo. Elas "Moldam e mudam o significado das atividades humanas: viajar significa quase sempre deslocar-se em algum veículo automotor; comunicar-se significa, cada vez mais, utilizar um telefone celular" (CUPANI, 2020, p. 84). Em certo sentido, ela está inerente em nossas atividades humanas.

Em uma outra dimensão, a tecnologia é compreendida por esse autor como uma atitude tecnológica. Essa dimensão é uma das mais difíceis de perceber, segundo o autor, pois se manifesta como uma determinada atitude ou propósito (ação tecnológica) do homem perante a realidade. Seria o modo de manifestação mais individualizado ou subjetivo dos sujeitos, uma maneira única, como a motivação da pessoa que se conecta com a produção, o uso e o conhecimento dos artefatos.

A partir do exposto, podemos considerar que a tecnologia não é vista apenas como um artefato tecnológico sofisticado ou como algo que esteja sempre relacionado ao futuro. Ela não é um objeto neutro, apresenta-se como um elemento de dimensão também interdisciplinar que modificou o nosso modo de vida desde a pré-história. Ela modifica, ainda, a nossa cultura. Indo além, ela possui conhecimentos específicos.

\section{Perspectiva crítica da tecnologia por Andrew Feenberg}

A Filosofia da Tecnologia vem tendo mais visibilidade nos estudos sobre Ciência e Tecnologia (C\&T) no contexto latino-americano a partir da Teoria Crítica da Tecnologia (GEREMIAS, 2016), que é defendida pelo filósofo Andrew Feenberg (2010).

Este autor problematiza e questiona a tecnologia por meio da ação e do pensamento crítico. Ele aponta que qualquer abordagem da tecnologia deve ir além das concepções e das interpretações das teorias deterministas, instrumentalistas e substantivas (essencialista).

$\mathrm{Na}$ concepção determinista, a tecnologia é descrita por ele como controlada humanamente. Ela é autônoma, é visualizada como uma ferramenta capaz de ser usada para qualquer fim, controlando os seres humanos e moldando a sociedade a uma exigência de eficiência e progresso (FEENBERG, 2010).

Já na perspectiva da teoria instrumentalista, para o mesmo autor, a tecnologia é neutra e controlada pelos desejos humanos. Nessa concepção, a tecnologia é vista somente para trazer benefícios à sociedade e ao seu progresso. Seus artefatos tecnológicos têm a mesma utilidade em qualquer outro contexto.

A vertente substantivista é associada ao determinismo tecnológico e ao essencialismo. Nessa concepção, ela não é simplesmente instrumental para qualquer valor (poder), pois já traz consigo certos valores (intrínsecos) triunfantes sobre todos os outros. Aqui, os meios e os fins encontram-se unidos em sistemas (FEENBERG, 2010). Essas concepções são, ainda 
segundo o autor, dominantes nos debates da mídia, no universo acadêmico e outros espaços.

Já a vertente intitulada de teoria crítica, e defendida por ele, incorpora e compartilha características de teorias anteriores, tendo em vista que "Concorda com o instrumentalismo que a tecnologia é controlável em algum sentido e também concorda com o substantivismo que a tecnologia está carregada de valores" (FEENBERG, 2010, p. 9).

Essa teoria reconhece ainda as consequências catastróficas que o desenvolvimento tecnológico tem causado. Feenberg (2010) atribui essas consequências à falta de capacidade humana para criar e para introduzir mecanismos de controles mais democráticos que possam reformular as tecnologias e o modo como nos relacionamos com ela. Nessa direção, o autor defende que é possível transformar a sociedade tecnológica em um lugar melhor para se viver.

Partindo desse entendimento, a tecnologia não é vista meramente como um artefato tecnológico (instrumento), como um objeto neutro, mas como uma possibilidade para o desenvolvimento de outros caminhos alternativos que tenham consigo uma visão crítica. É nessa perspectiva que os estudos CTS devem se pautar. A seguir, é apresentado o percurso metodológico da revisão de literatura.

\section{Metodologia}

Este estudo é caracterizado como um tipo de pesquisa bibliográfica. Segundo Gil (2008), esse tipo de pesquisa é desenvolvido com base em material já elaborado, constituído principalmente de livros e de artigos científicos.

A seleção dos artigos foi realizada durante o período de maio e junho do ano de 2021, e se configurou em cinco etapas: 1) Seleção dos termos de busca; 2) Seleção dos periódicos e das bases para estudo sistemático; 3) Seleção de artigos por meio de leitura flutuante; 4) Leitura aprofundada para análise do corpus; e 5) Criação de categorias a posteriori.

As palavras-chave utilizadas para a busca de artigos foram: "tecnologia", "ensino de ciências", "filosofia da tecnologia", "tecnologia e educação", "natureza da tecnologia"; "technology", "science teaching", "technology philosophy", "technology and education" e "nature of technology". A revisão de literatura compreendeu somente a busca de artigos do período entre 2010 a 2020. Utilizamos duas fontes de pesquisa, a saber: bases de dados ${ }^{3}$ e de periódicos ${ }^{4}$ qualificados pela Coordenação de Aperfeiçoamento de Pessoal de Nível

\footnotetext{
${ }^{3}$ Scielo; Academic Search Premier - ASP (EBSCO); Educational Resources Information Center (ERIC); Springerlink; Web Of Science - Coleção Principal (Clarivate Analytics); Wiley Online Library; Scopus; Academic Onefile (GALE). ${ }^{4}$ Ciência \& Educação; Cultural studies of Science education; Ensaio. Pesquisa em Educação em Ciências; Revista Brasileira de Educação; Enseñanza de la Ciencias; Journal of Science Education and Technology; Research in Science Education; Revista Eureka sobre Enseñanza y Divulgación de las Ciencias; Science \& Education; Science Education; ALEXANDRIA; Acta Scientiae: revista de ensino de ciências e matemática; Amazônia- Revista de Educação em Ciências e Matemática; Arete Revista Amazônia de Ensino de Ciências; Contexto \& Educação; Enseñanza de la Ciencias de la Tierra; Investigações em Ensino de Ciências; REEC. revista electrónica de Enseñanza de las Ciencias; RENCIMA - Revista de Ensino de ciências E Matemática; Revista brasileira de Ensino de Ciências e Tecnologia; Revista brasileira de Pesquisa em Educação em Ciências; Revista de Educação, Ciências e Matemática; Revista electrônica de Investigacion em Educación em Ciencias; Revista Eletrônica Ensino, Saúde e Ambiente; Revista Práxis; Alambique; Ciência e Ensino; Ciencia, docência y tecnologia; Eccos Revista Científica; Ensino de Ciências e Tecnologia em revista; Experiências em Ensino de Ciências; Góndola, Enseñanza Y
} 
Superior (CAPES) na área de Ensino de Ciências, nos idiomas Português, Espanhol e Inglês, que estivessem classificados no Qualis Quadriênio 2013-2016 em A1, A2, B1 e B2.

Para a inclusão dos artigos, foram empregados os seguintes critérios: artigos científicos que tratassem do tema tecnologia no ensino de Ciências e/ou Educação CTS no âmbito do Ensino Fundamental Anos Finais, Ensino Médio e Ensino Superior; pesquisas publicadas em periódicos de língua portuguesa, espanhola e inglesa, com textos disponíveis na íntegra e acesso gratuito.

Para a exclusão dos artigos foram empregados os seguintes critérios: estudos em que as pesquisas não fossem realizadas no ensino formal; artigos que apresentavam duplicidade entre as bases; estudos em outras línguas que não as escolhidas previamente; arquivos que não estivessem com textos disponíveis na íntegra, como resumos, resenha de livro, capítulos, referenciais com resumos e outras fontes.

Após seleção dos artigos por meio da leitura flutuante dos títulos, realizamos uma leitura aprofundada das publicações e emergiram as categorias a posteriori. Estas consistem em uma síntese de informações extraídas da pesquisa para representar os dados, são os elementos em comuns de significação de acordo com a análise de conteúdo de Bardin (1977).

Assim, emergiram três categorias: Natureza do Trabalho, Concepções de Tecnologia e Tecnologia na Abordagem CTS. Os resultados são apresentados a seguir.

\section{Resultados e discussões}

Considerando os critérios de inclusão adotados, foram selecionados 39 artigos científicos. De maneira geral, o resultado encontrado nesse levantamento indica que o tema não tem sido muito abordado durante a última década, ou seja, foi perceptível que é reduzido o número de trabalhos divulgados e publicados sobre esse assunto.

Podemos observar as publicações que envolveram a temática tecnologia no Ensino de Ciências e/ou Educação CTS nos últimos anos 2010-2020, como destaca a Figura 1.

Em conformidade com a figura apresentada, os estudos que envolveram a temática tecnologia no Ensino de Ciências vêm sendo publicados na literatura ainda de maneira pontual nos últimos anos. No total de 39 artigos selecionados, os resultados mostram que o número mais significativo dessa amostra aparece no ano de 2016, com 9 (23,07\%) artigos publicados. A seguir, serão discutidos os resultados agrupados nas categorias a posteriori estabelecidas para essa análise.

Aprendizaje de las Ciencias; RECEl - Revista Eletrônica Científica Ensino Interdisciplinar; Revista Ciências e Ideias;Revista Ciência e Tecnologia; Revista Educação e tecnologia; Revista tecnologias na educação; Science Education International; TEAR: revista de Educação, Ciência e Tecnologia; Revista de Educação Matemática e Tecnológica Iberoamericana; Revista Novas Tecnologias na Educação; Revista Iberoamericana de Tecnologia en Educación y Educación en Recnologia; Actio: Docência em Ciências; Conexões: Ciência e Tecnologia; Revista Ciência \& Ensino; Educação em Foco; Revista Internacional de Ciências (RIC); Revista de Educação em Ciências e Matemáticas; Ensino, Educação e Ciências Humanas; Indagatio Didactica. 


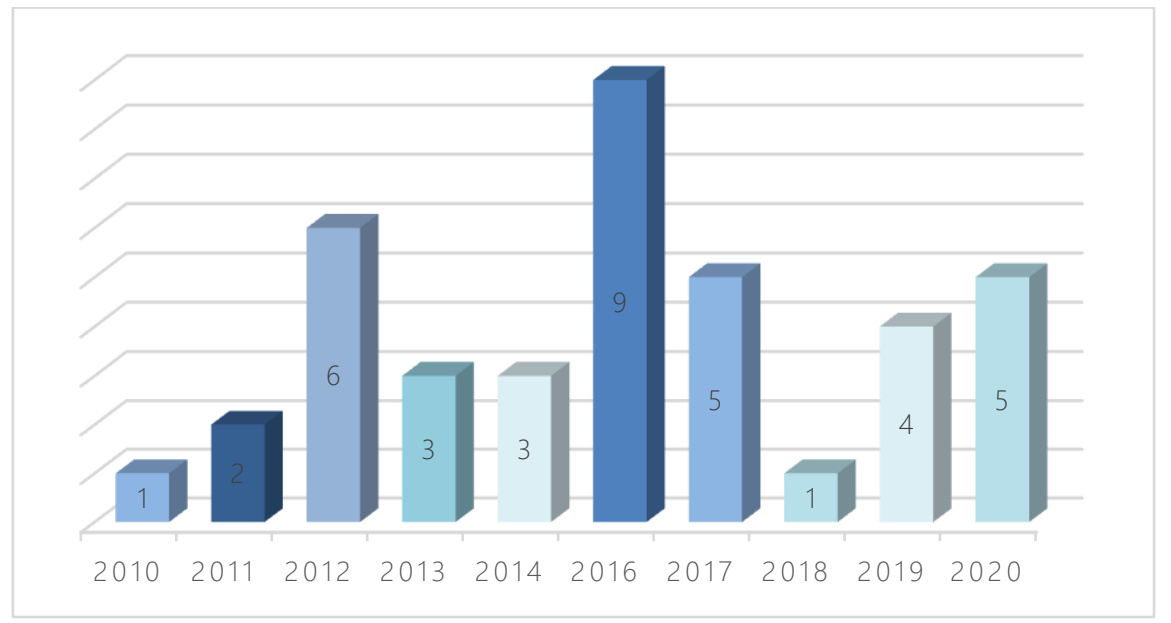

Figura 1: Mapeamento das produções entre 2010 e 2020

\section{Categoria Natureza do trabalho}

Por se tratar de pesquisas desenvolvidas para o público-alvo formação de professores e educação básica, buscamos identificar qual era a natureza dos artigos selecionados. A Figura 2 indica os resultados dessa categoria.

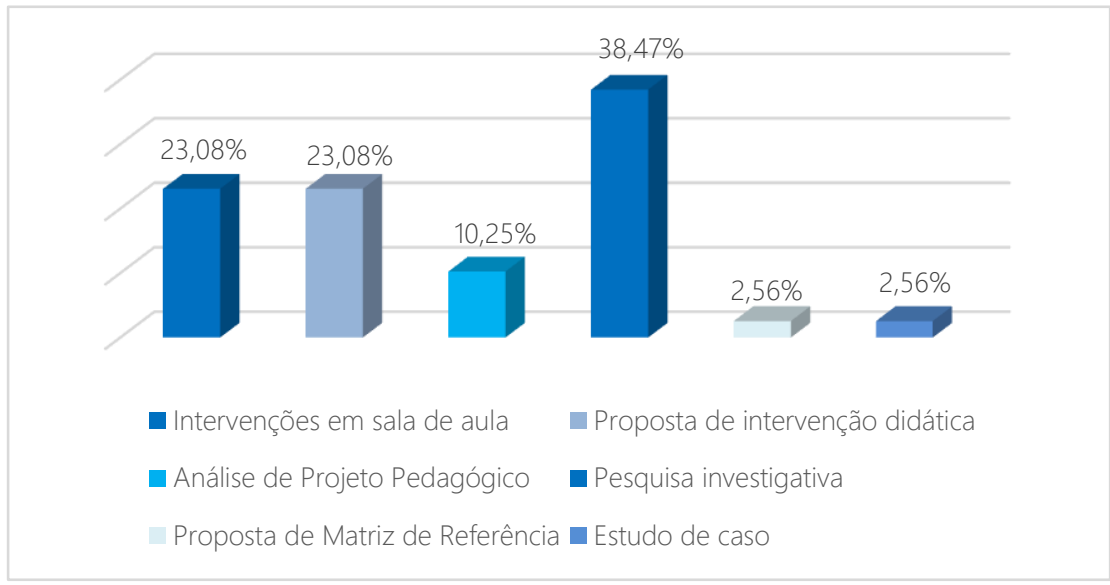

Figura 2: Natureza dos trabalhos analisados

De acordo com a figura 2, a maior parte das publicações que abordaram a tecnologia no Ensino de Ciências foram publicações de natureza investigativa, 15 (38,47\%) do total. Da análise, emergiram outras subcategorias: Intervenção em sala de aula e Proposta de intervenção didática, ambas com 9 (23,08\%); Análise de Projeto Pedagógico/Currículo, com 4 (10,25\%) produções; Estudo de caso e Proposta de Matriz de referência, ambas com 1 (2,56\%) estudo.

O artigo de Venessa e colaboradores (2019) é uma pesquisa descritiva e exploratória que buscou investigar a opinião de professores em formação, do curso de Química da Sriwijaya University - Indonésia, sobre a natureza da ciência e da tecnologia. Nessa pesquisa, os autores aplicaram um questionário, inspirados no questionário canadense Views On Science-Technology-Society (VOSTS) (AIKENHEAD et al., 1989). Esse questionário é considerado uma referência em trabalhos que investigam as concepções CTS. Nessa investigação, os autores inferiram que os pesquisados apresentaram declarações parcialmente consistentes com a visão geral da ciência. 
Outros estudos tiveram alguns pontos em comum, como as produções de Nunes e Dantas (2012) e de München, Tolentino-Neto e Adaime (2017), que aplicaram um questionário adaptado parcialmente do VOSTS aos professores em formação do curso de Química, a fim de analisar as compreensões de CTS/A. Nunes e Dantas (2012) realizaram a investigação na Universidade Estadual do Rio Grande do Norte (UERN). Em seus resultados, inferiram que os estudantes possuíam crenças positivas em relação à ciência e à tecnologia, advindas da influência do pensamento positivista.

Já München, Tolentino-Neto e Adaime (2017) aplicaram o questionário em uma Universidade pública da região centro-oeste do Brasil. Em seus resultados, encontraram uma diversidade de respostas fragmentadas em três concepções/categorias (simplistas, plausível, realista) sobre CTS, dentre elas, a categoria plausível se apresentou de forma mais acentuada.

A publicação de Santos, Tenório e Sunheimer (2018) analisa visões de ciência e tecnologia de licenciandos em Física da Universidade Federal Rural de Pernambuco (UFRPE), antes e após o contato com a robótica educacional. Em seus resultados, inferiram que, no pós-teste, os alunos apresentaram uma maior tendência para as visões menos tradicionais e mais contemporâneas da ciência e tecnologia.

Já Deconto, Cavalcanti e Ostermann (2016) analisaram as compreensões de licenciandos em Física da Universidade Federal do Rio Grande do Sul, sobre as interrelações CTS a luz da teoria Bakhtiniana e pressupostos da Educação CTS de Srieder (2012). $\mathrm{Na}$ investigação, inferiram que as compreensões dos estudantes foram confusas e ambíguas, marcadas por um grau do senso comum.

No artigo dos autores Malta, Dorvillé e Galieta-Nascimento (2020), foi analisada a visão de 12 licenciandos em Ciências Biológicas de uma universidade pública do Rio de Janeiro por meio do método grupo focal. Em seus resultados, pontuaram que os estudantes apresentaram concepções positivas sobre C\&T. Porém, no decorrer da atividade, eles convergiram para posturas mais críticas a respeito do tema.

Nesse levantamento, observamos que apenas um estudo teve como objetivo principal investigar as concepções de C\&T de professores universitários. Trata-se do artigo dos autores Erlam e Morales (2017). Eles entrevistaram um grupo de professores do departamento de Física, Química e Biologia da Faculdade de Ciências Básicas da Universidade de Tolima - Colombia. Em seus resultados, observaram que os professores em sua maioria compreendiam a tecnologia como uma ferramenta da ciência. E, destes, apenas um forneceu um conceito mais adequado e atualizado sobre a natureza da tecnologia.

O estudo de Pavón e Fernández (2017) apresenta resultados semelhantes. Em sua investigação, realizada com cinco professores Chilenos de Química do Ensino Médio, foi identificado que eles possuem concepções ingênuas sobre a natureza da ciência e tecnologia, principalmente por incorporarem o sentido da tecnologia a ciência.

A investigação de Waight (2013), realizada com 30 professores de ciências do Ensino Médio, em uma cidade do nordeste dos Estados Unidos da América (EUA), foi identificado que a definição dada a tecnologia para eles era o artefato. Os entrevistados ainda atribuíram à tecnologia uma perspectiva salvacionista e algo que representava o avanço das civilizações.

Outras publicações realizaram suas investigações a partir de livros - Oliveira e Gonçalves (2019); Macedo e Silva (2010) - e de currículo - Alonso e Manassero (2012b). Os autores Oliveira e Gonçalves (2019) identificaram que a obra denominada Usina (1936), do 
autor brasileiro José Lins do Rego, tem um alto valor pedagógico a ser desenvolvido em uma perspectiva crítica de Educação CTS na educação básica.

Na subcategoria Intervenção em sala de aula, Geremias e Cassiani (2016) realizaram uma oficina (animação/educativa) sobre a produção do artefato papel, com professores em formação de ciências/biologia integrantes do grupo Observatório da Educação (OBEDUFSC - Ciências), da Universidade Federal de Santa Catarina (UFSC), a fim de mobilizar outros sentidos sobre tecnologia.

Rehmat e Bailey (2014) integraram a tecnologia em curso de métodos de ciências elementares, em uma universidade no sudoeste dos EUA, com professores de formação inicial. Nesse curso, eles buscaram compreender a interação da tecnologia no ensino de ciências na visão dos estudantes.

Outras intervenções realizadas no ensino superior foram as publicações de Freitas e Queirós (2020); e Sepine e Maciel (2016). Os autores Freitas e Queirós (2020) realizaram uma sequência didática em que buscaram compreender as concepções das interações CTS de sete licenciandos do curso de Física, da Universidade Federal de Mato Grosso do Sul (UFMS). Em um questionário realizado antes e após as ações, foram reconhecidos, por parte da maioria dos licenciandos, os mitos relacionados à C\&T. Os autores sinalizaram a necessidade de novas intervenções didáticas.

Já Sepine e Maciel (2016) realizaram uma intervenção pedagógica estruturada sob a temática Natureza da Ciência e Tecnologia, através de um curso de formação inicial de Ciências Biológicas, no Instituto Federal de Minas Gerais (IFMG), campus Machado-MG, com uma carga horária de 30h. Em seus resultados, inferiram que intervenções didáticas dessa natureza possibilitam alcançar um resultado mais amplo ao que se refere aos conhecimentos conceituais, procedimentais e atitudinais, além de contribuir para a formação cidadã dos futuros professores.

Ao que se refere à formação continuada de professores, encontramos apenas um estudo na literatura internacional. Pavón, Soto e Fernàndez (2019) relatam uma sequência de atividades realizadas com professores de Química, no Chile. Nos resultados, é apontado que a intervenção proporcionou uma maior reflexão sobre a natureza da ciência e tecnologia.

Os autores Quevado e Puente (2019); Ribeiro, Colherinhas e Genovese (2016); Oliveira, Guimarães e Lorenzetti (2016); Sepini, Alonso e Maciel (2014) realizaram suas intervenções didáticas na educação básica.

Quevado e Puente (2019) realizaram uma oficina introdutória com turmas de ciências naturais do $6^{\circ}$ ano do ensino básico da Segóvia. Na sequência didática, eles trabalharam o conteúdo curricular energia e quatro temas relacionados à natureza da ciência e tecnologia, a fim de promover uma contextualização e uma visão não distorcida da ciência e tecnologia.

Ribeiro, Colherinhas e Genovese (2016) relatam uma experiência a partir da temática CTS/A, desenvolvida no ensino médio de uma escola pública de Goiânia-GO. Nas atividades, eles deram ênfase a temas ligados a diferentes aparelhos tecnológicos, como: rádio AM/FM; televisão; aparelho de raio-x; radioterapia; celular; telescópio; microscópico entre outros, que fossem relacionados a espectro eletromagnético e ao conteúdo - ótica e ondas - do currículo de Física. A experiência proporcionou um maior envolvimento por parte dos alunos e mobilizou uma discussão, entre os envolvidos, sobre a tecnologia. 
Em uma escola da rede pública de ensino médio da região metropolitana de CuritibaPR, os autores Oliveira, Guimarães e Lorenzetti (2016) analisaram as concepções de tecnologia apresentadas por alunos durante uma intervenção didático-pedagógica com Enfoque CTS. Ao final, eles pontuam que as atividades proporcionaram maior participação e maior interesse sobre o assunto abordado.

A produção de Sepini, Alonso e Maciel (2014) relata uma intervenção didática/experimental, que partiu de temas da natureza da ciência e tecnologia em uma escola localizada no sul do Estado de Minas Gerais. Os autores inferiram que as atividades desenvolvidas possibilitaram aos estudantes alcançar uma mudança nas concepções atitudinais acerca da natureza da ciência e tecnologia.

Ao que se refere à subcategoria Proposta de intervenção didática, alguns autores propõem estratégias didáticas para o ensino da natureza da ciência e tecnologia e Educação CTS em seus textos (FREDERIK; SONNEVELD; VRIES, 2011; KRUSE; WILCOX, 2013; DÍAZ; CARMONA, 2016; FIRME, 2020; SANTILLI, 2012; HADJILOUCA; CONSTANTINOU; PAPADOURIS, 2011; RESTREPO; PARADA; PORRAS, 2012; SILVA, 2013; ERLAM; OLIVEROS, 2012).

Frederik, Sonneveld e Vries (2011), em seu estudo, abordam a filosofia da tecnologia para desenvolver uma conceituação de artefatos técnicos que podem ser usados para fins educacionais no ensino de Ciências.

Já Díaz e Carmona (2016) descrevem uma proposta de ensino direcionada à formação de futuros professores do Ensino de Ciências sobre a Natureza da Tecnologia. Firme (2020) analisa uma proposta de organização de conteúdo para a abordagem CTS no ensino de Química. A autora ainda buscou caracterizar do ponto de vista filosófico a tecnologia nessa abordagem.

Em uma outra publicação, o referido autor Santilli (2012) descreve três estudos de caso históricos, visando contribuir para que professores e alunos compreendessem melhor a interdependência entre a ciência e a tecnologia, e a forma como elas se relacionam com a sociedade, bem como proporcionar uma visão geral da natureza da ciência e tecnologia.

Hadjilouca, Constantinou e Papadouris (2011) propõem uma sequência didática direcionada a estudantes do Ensino Fundamental e Médio, a fim de apreciar a interrelação da ciência e tecnologia. Para desenvolver essa sequência, eles se apoiaram nas fontes: a história e a filosofia da ciência e tecnologia, conhecimento sobre o ensino e aprendizagem da natureza da ciência e tecnologia, dados empíricos sobre as dificuldades e as ideias iniciais dos alunos. A temática proposta parte do contexto de lentes e instrumentos ópticos, dividida em três unidades didáticas: 1) processo de design tecnológico; 2) investigação científica; 3) distinções e relações entre ciência e tecnologia.

Direcionadas ao ensino fundamental anos finais Restrepo, Parada e Porras (2012) propõem duas unidades didáticas, denominadas: "Uma conversão diária de energia. Até onde vamos em sua análise?" e "metabolismo energético", no intuito de melhorar a compreensão da natureza da ciência e da tecnologia de alunos da educação básica colombiana. Já Silva (2013) propõe uma sequência de ensino que procura discutir elementos, desde o surgimento, desenvolvimento e funcionamento, do artefato telefone.

Erlam e Oliveros (2012) descrevem a construção de unidades didáticas com a temática estatuto epistemológico da ciência, para professores em formação de ciências da Universidade Del Tolima, Colômbia, com o objetivo de contribuir para a reflexão sobre o processo de intervenção didática no âmbito da natureza da ciência e tecnologia. 
Na subcategoria Análise de Projeto Pedagógico/Currículo, o estudo de Dominiano e Lorenzetti (2020) analisa as abordagens da Educação CTS presentes no Projeto Pedagógico de Curso (PPC) de Licenciatura em Ciências da Universidade Federal do Paraná (UFPR), Litoral, na percepção e na prática dos docentes formadores. Nesse estudo, foi constatado que as abordagens da Educação CTS crítica estavam presentes no fazer docente dos professores formadores.

Alonso e Manassero (2012a) analisam currículos de ciências e a competência científica do Programa Internacional de Avaliação de Alunos (PISA). Eles identificam os conteúdos para o ensino da natureza das ciências e da tecnologia presentes nos currículos espanhóis do ensino secundário e médio, e ainda propõem um desenho de um quadro curricular para esses temas.

Ricardo (2020) analisa dezenove PPC's de Licenciatura em Física de Instituições públicas de Ensino Superior do Estado de São Paulo. Em suas análises, ele inferiu que os PPC's indicam concepções de tecnologia como ciência aplicada, como artefatos, como modelo linear de desenvolvimento tecnológico e determinismo tecnológico. Em concordância com esse autor, sinalizamos a necessidade dos cursos de Ensino Superior de professores de ciências reverem o sentindo de tecnologia e da sua relação com a ciência e com os aspectos sociais, históricos, econômicos e políticos nos mais variados contextos.

Nesse sentindo, é certo que os cursos de formação de professores precisam superar as concepções simplificadas de tecnologia, a fim de proporcionar uma concepção ampliada e profunda desse conceito, tal apontamento poderia ser um dos objetivos desses cursos.

Na última subcategoria, Bispo-Filho, Maciel e Cabral (2016) propõem uma proposta de matriz curricular com enfoque em natureza da ciência e tecnologia, na tentativa de potencializar a avaliação de competências científicas. Em suas considerações finais, os autores sinalizam a necessidade de discutir essa temática na formação docente.

\section{Categoria Concepções de Tecnologia}

Essa categoria emergiu após a leitura dos artigos de natureza investigativa, análise curricular e intervenção didática. Nesses trabalhos, foi observado que, em seus resultados, os dados sobre as concepções de tecnologia convergiam. Esses artigos tinham como amostra/participantes de pesquisa professores em exercício, professores em formação e/ou estudantes da educação básica.

Quadro 1: Subcategorias construídas

\begin{tabular}{|c|l|}
\hline $\begin{array}{c}\text { SUnCATEGORIAS } \\
\text { Conções reduzidas } \\
\text { de tecnologia }\end{array}$ & $\begin{array}{l}\text { Incorpora a concepção de tecnologia, como: salvacionista, ciência } \\
\text { aplicada, neutra, instrumentalista, determinista e substantivista. }\end{array}$ \\
\hline $\begin{array}{c}\text { Concepções críticas da } \\
\text { tecnologia }\end{array}$ & $\begin{array}{l}\text { São concepções de tecnologia a partir de outras dimensões, com } \\
\text { um viés crítico. Seriam concepções que se assumem como um } \\
\text { conhecimento tecnológico, não neutro, que possui articulação com } \\
\text { questões políticas, econômicas, ambientais e éticas; e também como } \\
\text { uma percepção de que os artefatos tecnológicos podem modificar a } \\
\text { nossa cultura. }\end{array}$ \\
\hline
\end{tabular}


Identificadas as unidades de registro, separamos as concepções de tecnologia em duas subcategorias (Quadro 1) que construímos baseadas nos descritos dos autores Bazzo, Linsingen e Pereira (2003) Feenberg (2010); Bazzo (2014) e Cupani (2016).

Após análise, foi observado que a maioria dos resultados das investigações trazia enxertos que iam ao encontro de concepções reduzidas de tecnologia, como os artigos dos autores Rehmat e Bailey (2014); Nunes e Dantas (2012); Erlam e Oliveros (2017); Pavón e Fernández (2017); Waight (2013); Deconto, Calvanti e Ostermann (2016), dentre outros. Podemos observar alguns enxertos a seguir, no Quadro 2.

Quadro 2: Concepções reduzidas de tecnologia

\begin{tabular}{|c|c|}
\hline AUTOR(ES) & ENXERTOS \\
\hline $\begin{array}{l}\text { REHMAT; } \\
\text { BAILEY, 2014, p. } \\
755\end{array}$ & $\begin{array}{l}\text { "Tecnologia é qualquer coisa eletrônica, computadores, iPads, iPods, placas } \\
\text { SMART, telefones celulares, etc. Eles são usados pela maioria das pessoas na } \\
\text { escola e em casa". }\end{array}$ \\
\hline $\begin{array}{l}\text { FREDERIK; } \\
\text { SONNEVELD; } \\
\text { VRIES, 2011, p. } \\
1141 .\end{array}$ & $\begin{array}{l}\text { "As definições de tecnologia revelaram ênfase na tecnologia como artefato, } \\
\text { otimismo esmagador sobre o propósito e a função da tecnologia para } \\
\text { melhorar e tornar a vida mais fácil e como representação do avanço da } \\
\text { civilização". }\end{array}$ \\
\hline $\begin{array}{l}\text { (ERLAM; } \\
\text { OLIVEROS, } \\
2017, \text { p. } 11 .\end{array}$ & $\begin{array}{l}\text { "Na explicação sobre a natureza da tecnologia, eles não a separam da ciência, } \\
\text { mas, por sua vez, eles erroneamente consideram isso como dependente e } \\
\text { ferramenta a serviço da ciência". }\end{array}$ \\
\hline $\begin{array}{l}\text { RICARDO, } \\
2020, \text { p. } 203 .\end{array}$ & $\begin{array}{l}\text { "À redução da tecnologia a uma simples aplicação da ciência, ou ao artefato } \\
\text { tecnológico, foram as concepções mais comuns encontradas em todos os } \\
\text { Projetos investigados". }\end{array}$ \\
\hline $\begin{array}{l}\text { DECONTO; } \\
\text { CAVALCANTI; } \\
\text { OSTERMANN, } \\
\text { 2016, p. } 115\end{array}$ & $\begin{array}{l}\text { "Os licenciandos apresentam uma postura que considera a tecnologia como } \\
\text { algo que deriva dos conhecimentos científicos [...] não se verifica a ideia de } \\
\text { conceber a tecnologia como um processo, apenas como um produto. [...] } \\
\text { incluem uma visão alinhada à perspectiva salvacionista da ciência e da } \\
\text { tecnologia". }\end{array}$ \\
\hline $\begin{array}{l}\text { PAVÓN; } \\
\text { FERNÁNDEZ, } \\
\text { 2017, p. } 33 .\end{array}$ & $\begin{array}{l}\text { "À incorporação da palavra tecnologia foi um desafio para os professores, } \\
\text { cujas respostas sempre se referiam à ciência". }\end{array}$ \\
\hline
\end{tabular}

A partir desses enxertos, é possível notar uma concepção de tecnologia reduzida, direcionada apenas a artefatos tecnológicos, uma ciência aplicada e a presença de uma percepção linear de desenvolvimento. Alinhados a uma perspectiva salvacionista, a ciência gera tecnologia, que, juntas, produzem desenvolvimento econômico e social (BAZZO, LINSINGEN; PEREIRA, 2003).

Ainda nessa subcategoria, foi percebida a predominância da corrente (pensamento) instrumentalista. Segundo Feenberg (2010), essa é a concepção de senso comum para a maioria das pessoas. Nessa perspectiva de tecnologia, os sujeitos compreendem que elas são apenas ferramentas à disposição para servir os seus próprios propósitos. Aqui, ela é neutra, isenta de valores e controlada pelos seres humanos em busca de benefícios e de progresso da sociedade.

Outros estudos mencionaram que os professores em formação e/ou estudantes da educação básica se aproximavam de uma visão ampliada de tecnologia, após a realização de intervenções/sequências didáticas (MALTA; DORVILLÉ; GALIETA-NASCIMENTO, 2020; 
KRUSE; WILCOX, 2013; RIBEIRO; COLHERINHAS; GENOVESE, 2016; OLIVEIRA; GUIMARÃES; LORENZETTI, 2016; SEPINI; ALONSO; MACIEL, 2014; SANTOS; TENÓRIO; SUNDHEIMER, 2018; ALONSO; MANOSSERO, 2013).

Essas pesquisas tinham como objeto de estudo diferentes abordagens, dentre elas: natureza da ciência e tecnologia; abordagens CTS em uma perspectiva crítica e abordagens CTS com o enfoque para a tecnologia.

Assim, por mais que seja complexo trabalhar a tecnologia no sentindo amplo, apontamos a necessidade de mais professores e de mais pesquisadores desenvolverem ações de projetos de ensino interdisciplinares, de sequências didáticas e/ou de intervenções pedagógicas que partam da perspectiva de educação CTS crítica.

Essas ações podem trazer resultados positivos e uma compreensão do que seria tecnologia em seu sentido amplo, como foi inferido nos resultados dos autores supracitados.

\section{Categoria Tecnologia na Abordagem CTS}

Essa categoria emergiu após identificarmos os artigos que assumiram em seus referenciais pressupostos da abordagem CTS. Após a leitura dos mesmos, foi observado que em grande maioria não é dado uma maior ênfase ao elemento Tecnologia na tríade CTS. Essa ratificação vai de encontro com a sinalização feita por alguns autores: GalietaNascimento e Linsingen (2006); Bocheco (2011); Cassiani et al. (2014); Geremias (2021); e Lorenzetti (2021).

Quadro 3: Enxertos do silenciamento da tecnologia na abordagem CTS

\begin{tabular}{|l|l|}
\hline \multicolumn{1}{|c|}{ AUTORES } & \multicolumn{1}{c|}{ EXCERTOS } \\
\hline $\begin{array}{l}\text { STRIEDER; TORIJA; } \\
\text { QUILEZ, 2017, 44. }\end{array}$ & $\begin{array}{l}\text { "O desenvolvimento tecnológico é o parâmetro menos abordado na } \\
\text { Edução CTS" [...] "Apesar da sua importância, ainda é relegada a segundo } \\
\text { lugar na formação científica dos alunos". }\end{array}$ \\
\hline $\begin{array}{l}\text { ABREU; STRIDER, } \\
\text { 2016, p. 885. }\end{array}$ & $\begin{array}{l}\text { "Espera-se que este estudo possa contribuir para que, na tríade CTS, o T } \\
\text { possa ter uma maior integração com o C e o S da sigla, evitando, assim, } \\
\text { umação da tecnologia ao status de ferramenta e/ou de ciência aplicada". }\end{array}$ \\
\hline $\begin{array}{l}\text { DORINIANO; } \\
\text { 2020, p. 13. }\end{array}$ & $\begin{array}{l}\text { "Isto pode ser decorrente da pouca atenção dada à tecnologia na educação } \\
\text { em ciências e, até mesmo, em abordagens CTS". }\end{array}$ \\
\hline $\begin{array}{l}\text { GEREMIAS; } \\
\text { CASSIANI, 2016, p. } \\
\text { 744. }\end{array}$ & $\begin{array}{l}\text { "Alguns pesquisadores criticam a pouca atenção dada ao campo tecnológico } \\
\text { na ETS [...] apesar da presença do T no centro do acrônimo". }\end{array}$ \\
\hline $\begin{array}{l}\text { OLIVEIRA; } \\
\text { GONÇALVES, } \\
\text { 2019, p. 19. }\end{array}$ & $\begin{array}{l}\text { "O livro Usina possibilita a abordagem de conteúdos relacionados às } \\
\text { interaçes CTS, valorizando inclusive a dimensão tecnológica, nem sempre } \\
\text { privilegiada nas discussões de caráter CTS no ensino de ciências da natureza". }\end{array}$ \\
\hline
\end{tabular}

Outro aspecto importante a ser constatado foi que cerca de 7 (18,42\%) publicações destacaram haver um silenciamento da tecnologia nas abordagens CTS (GEREMIAS; CASSIANI, 2016; DOMINIANO; LORENZETTI, 2020; OLIVEIRA; GONÇALVES, 2019; FIRME, 2020; STRIEDER; TORIJA; QUILEZ, 2017; OLIVEIRA; GUIMARÃES; LORENZETTI, 2016; ABREU; STRIEDER, 2016). Podemos observar alguns dos enxertos a seguir, no Quadro 3. 
Esse silenciamento pode acarretar em concepções reduzidas de tecnologia. Por isso, frisamos a urgente necessidade de um tratamento mais adequado dado à tecnologia nos cursos de formação inicial e continuada de professores de Ciências.

Nesse sentindo, enfatizamos como necessária a disseminação de reflexões que se originem de um referencial teórico crítico sobre tecnologia, pois elas possibilitam a abertura para um olhar interdisciplinar e contextualizado para com a Tecnologia, bem como sua relação com os demais elementos ciência e sociedade (C e S) da abordagem CTS no ensino de Ciências.

Concordamos com Oliveira, Guimarães e Lorenzetti (2016), quando descrevem que é imprescindível que as abordagens CTS deem igual importância ao elemento Tecnologia (T) em relação aos demais elementos ciência e sociedade ( $C$ e S), pois é fundamental a implantação de práticas em sala de aula que oportunizem condições para que os estudantes possam analisar os diferentes aspectos que caracterizam o desenvolvimento tecnológico.

\section{Considerações finais}

Os resultados apresentados neste estudo fazem parte do recorte de uma pesquisa de dissertação de mestrado acadêmico desenvolvida através do Programa de Pós-Graduação em Ensino de Ciências e Matemática (PPGECM) da Universidade Federal do Rio Grande do Norte (UFRN).

A revisão de literatura apresentada buscou descrever o mapeamento das publicações que trabalharam o elemento (T) na Educação CTS no/ou ensino de Ciências, a fim de evidenciar pesquisas realizadas nesse campo. De uma forma geral, podemos dizer que a ênfase dada à tecnologia nesse campo de estudo vem surgindo de maneira pontual nos últimos anos, sendo ainda mais incipiente nas pesquisas brasileiras. Por se tratar de uma temática pouco usual de pesquisa, inferimos a necessidade de maior discussão da tecnologia nesse campo de estudo.

Nesse estudo, ainda foi percebido que a maioria dos resultados das pesquisas trazem concepções restritas (simplistas) de tecnologia, seja de professores em exercício, professores em formação ou estudantes da educação básica. As concepções identificadas em nossos resultados aludem ao entendimento da tecnologia como instrumentalista, a artefatos tecnológicos, ciência aplicada e ligada a uma presença de uma percepção linear de desenvolvimento.

Nesse sentido, sinalizamos como alternativas, para que ocorra a assunção da tecnologia no sentido mais amplo, a realização de intervenções didáticas de Educação CTS crítica e a apresentação da natureza da tecnologia durante a formação inicial e continuada de professores de Ciências, uma vez que tais ações podem promover efeitos positivos que contribuam para a construção de uma concepção mais ampliada de tecnologia.

Por fim, consideramos como necessário que a tecnologia seja refletida e difundida em seu sentindo amplo, crítico e como produtora de conhecimentos específicos. Abordagens que partem desse entendimento no Ensino de Ciências estabelecem de fato uma efetiva educação científico-tecnológica. 


\section{Agradecimentos}

Agradecemos a Coordenação de Aperfeiçoamento de Pessoal de Nível Superior (CAPES) pelo apoio na realização dessa pesquisa.

\section{Referências}

ABREU, Rosana Oliveira Dantas; STRIEDER, Roseline Beatriz. Abordagens sobre tecnologia em trabalhos com enfoque CTS no ensino de Química no Brasil. Indagatio Didactica, v. 8, n. 1, p. 870-886, 2016.

ACEVEDO, Germán Darío Rodríguez. Ciencia, Tecnología y Sociedad: una mirada desde la Educación en Tecnología. Organización de Estados Iberoamericanos para la Educación, la Ciencia y la Cultura, 1998.

AIKENHEAD, Glen et al. Views on science-technology-society. Social Science and Humanities Research Council, 1989.

ALONSO, Ángel Vázquez; MANASSERO-MAS, María Antonia. La selección de contenidos para enseñar naturaleza de la ciencia y tecnología (parte 1): Una revisión de las aportaciones de la investigación didáctica. 2012. Revista Eureka sobre Enseñanza y Divulgación de las Ciencias, v. 9, n. 1, p. 630-648, 2012a.

ALONSO, Vázquez Ángel; MANASSERO-MAS, María Antonia. La selección de contenidos para enseñar naturaleza de la ciencia y tecnología (parte 2): Una revisión desde los currículos de ciencias y la competencia PISA. Revista Eureka sobre Enseñanza y Divulgación de las Ciencias, v. 9, n. 1, p. 32-53, 2012b.

ALONSO, Ángel Vázquez; MANASSERO-MAS, María Antonia. La comprensión de un aspecto de la naturaleza de ciencia y tecnología: Una experiencia innovadora para profesores en formación inicial. Revista Eureka sobre Enseñanza y Divulgación de las Ciencias, v. 10, p. 630648, 2013.

BARDIN, Lawrence. Análise de conteúdo. Lisboa: edições, v. 70, p. 225, 1977.

BAZZO, Walter Antonio; LINSINGEN, Irlan. Von; PEREIRA, Luiz Teixeira do Vale. (Org.). Introdução aos estudos CTS (ciência, tecnologia e sociedade). Florianópolis: Organização dos Estados Ibero-americanos Para A Educação, A Ciência e a Cultura (OEI), 2003.

BAZZO, Walter Antonio. Ciência, tecnologia e sociedade: e o contexto da educação tecnológica. 4 ed. Florianópolis: Editora da UFSC, 2014.

BOCHECO, Otávio. Parâmetros para a abordagem de evento no enfoque CTS. $165 f$. Dissertação (Mestrado em Educação Científica e Tecnológica). - Universidade Federal de Santa Catarina, Florianópolis, 2011.

BISPO-FILHO, Djalma Oliveira; MACIEL, Maria Delourdes; CABRAL, Sonia Aparecida. Uma matriz de referência para o Ensino da Natureza da Ciência e Tecnologia (NDC\&T) centrada na perspetiva Ciência Tecnologia e Sociedade (CTS). Revista de Ensino de Ciências e Matemática, v. 7, n. 1, p. 19-37, 13 fev. 2016.

CASSIANI, Suzani et al. O grupo DICiTE-discursos da ciência e da tecnologia na educação. Ciência \& Ensino (ISSN 1980-8631), v. 3, n. 1, p. 1-19, 2014. 
CUPANI, Alberto. Modalidades da tecnologia e suas consequências culturais. Revista Dialectus, Fortaleza, ano 9, n. 17, p. 82-95, maio/ago. 2020.

CUPANI, Alberto. Filosofia da tecnologia: um convite. 3. Ed. Florianópolis: Editora da UFSC, 2016.

DECONTO, Diomar Caríssimo Selli; CAVALCANTI, Cláudio José Holanda; OSTERMANN, Fernanda. A perspectiva ciência, tecnologia e sociedade na formação inicial de professores de física: estudando concepções a partir de uma análise bakhtiniana. Alexandria: Revista de Educação em Ciência e Tecnologia, v. 9, n. 2, p. 87-119, 2016.

DÍAZ, José Antonio Acevado; CARMONA, Antonio Gárcia. Una controversia de la Historia de la Tecnología para aprender sobre Naturaleza de la Tecnología: Tesla vs. Edison-La guerra de las corrientes. Enseñanza de las ciencias: revista de investigación y experiencias didácticas, 34 (1), 193-209., 2016.

DOMICIANO, Tamara Dias; LORENZETTI, Leonir. A educação ciência, tecnologia e sociedade no curso de licenciatura em ciências da UFPR litoral. Ensaio Pesquisa em Educação em Ciências (Belo Horizonte), v. 22, 2020.

ERLAM, Néstor Cardoso; MORALES, Edna Eliana Oliveros. Concepciones de tecnología en docentes universitarios de ciencias. Revista Científica, v. 3, n. 30, p. 195-206, 2017.

ERLAM, Néstor Cardoso; OLIVEROS, Edna. Eliana Morales. Unidad didáctica para la enseñanza de un tópico denaturaleza de la Ciencia y la Tecnología (ndcyt) en profesores de Ciencias en formacion de la Universidad del Tolima(ut)-Colombia, en el marco del proyecto eancyt1. Revista de Ensino de Ciências e Matemática, v. 3, n. 3, p. 91-104, 2012.

FEENBERG, Andrew. O que é a filosofia da tecnologia. Andrew Feenberg: racionalização democrática, poder e tecnologia, v. 3, p. 39-51, 2010.

FIRME, Ruth Nascimento. Abordagem ciência-tecnologia-sociedade (CTS) no ensino de ciências: de qual tecnologia estamos falando desde esta perspectiva em nossa prática docente? Góndola, enseñanza y aprendizaje de las ciencias, v. 15, n. 1, p. 65-82, 2020.

FREDERIK, Ineke; SONNEVELD, Wim; VRIES, Marc J. Teaching and learning the nature of technical artifacts. International journal of technology and design education, v. 21, n. 3, p. 277290, 2011.

FREITAS, Wélica Patrícia Souza; DE QUEIRÓS, Wellington Pereira. O processo de compreensão das interações ciência-tecnologia-sociedade (CTS) em um curso de formação inicial de professores de Física. Experiências em Ensino de Ciências, v. 15, n. 02, p. 324-347, 2020.

GEREMIAS, Bethania Medeiros. Produção de sentidos sobre tecnologia no grupo observatório da educação-ciências: discursos e problematizações. 2016. 303f. Tese (Doutorado em Educação Científica e Tecnológica). - Universidade Federal de Santa Catarina, Florianópolis, 2016.

GEREMIAS, Bethania Medeiros. Sentidos de Tecnologia nas pesquisas acadêmicas: olhares e compreensões na Área de Educação Científica e Tecnológica. In: PINHÃO, Francine Lopes et al. (Org.). Conversas com a Ciência: cadernos temáticos educação e Ciências da Natureza. Rio de Janeiro: Faculdade de Formação de Professores, 2021. Cap. 2, p. 25-39. 
GEREMIAS, Bethania Medeiros; CASSIANI, Suzani. A tecnologia como problema na educação em ciência, tecnologia e sociedade: análises de uma oficina de leitura na formação de professores de ciências. Inter-Acao, v. 41, n. 3, 2016.

GIL, Antônio Carlos. Como elaborar projetos de pesquisa. 4. ed. São Paulo: Atlas, 2008.

GALIETA-NASCIMENTO, Tatiana; VON LINSINGEN, Irlan. Articulações entre o enfoque CTS e a pedagogia de Paulo Freire como base para o ensino de ciências. Convergência, v. 13, n. 42, p. 95-116, 2006.

HADIILOUCA, Rodothea; CONSTANTINOU, Constaninos; PAPADOURIS, Nicos. The rationale for a teaching innovation about the interrelationship between science and technology. Science \& Education, v. 20, n. 10, p. 981-1005, 2011.

KRUSE, Jerrid.; WILCOX, Jesse. Engaging students with the nature of science and the nature of technology by modeling the work of scientists. The Clearing House: A Journal of Educational Strategies, Issues and Ideas, v. 86, n. 3, p. 109-115, 2013.

LORENZETTI, Leonir. [Aula Aberta - Sequencias didáticas e Educação CTS: implicações na formação do educando]. 2021. 1 vídeo (1h56m). Publicado pelo canal Liquens UERJ. Disponível em: https://www.youtube.com/watch?v=WzwsfsXcUqQ. Acesso em: 09 mai. 2021.

MACEDO, Cristina Cândida; SILVA, Luciano Fernandes. Contextualização e visões de ciência e tecnologia nos livros didáticos de física aprovados pelo PNLEM. Alexandria: Revista de Educação em Ciência e Tecnologia, v. 3, n. 3, p. 1-23, 2010.

MALTA, Filipe Lima; DORVILLÉ, Luís Fernando Marques; GALIETA-NASCIMENTO, Tatiana. Alfabetização científica e enfoque CTS na visão de licenciandos em ciências biológicas: uma análise de grupo local. Investigações em Ensino de Ciências, v. 25, n. 2, 2020.

MITCHAM, Carl. Thinking through technology: The path between engineering and philosophy. University of Chicago Press, 1994.

MÜNCHEN, Sinara; TOLENTINO-NETO, Luiz Caldeira Brant; ADAIME, Martha Bohrer. Compreensões de licenciandos em Química sobre as interações entre ciência-tecnologiasociedade. Revista Brasileira de Ensino de Ciência e Tecnologia. V. 10, n. 2, p. 132-152, 2017.

NUNES, Albino Oliveira; DANTAS, Josivania Marisa. As relações ciência-tecnologiasociedade-ambiente (CTSA) e as atitudes dos licenciandos em química. Educación química, $v$. 23, n. 1, p. 85-90, 2012.

OLIVEIRA, Daiane Quadros; GONÇALVES, Fábio Peres. Usina: articulações entre ensino, literatura e interações entre ciência, tecnologia e sociedade. Ensaio Pesquisa em Educação em Ciências, v. 21, 2019.

OLIVEIRA, Silvaney; GUIMARÃES, Orliney Maciel; LORENZETTI, Leonir. O enfoque CTS e as concepções de tecnologia de alunos do ensino médio. Alexandria: Revista de Educação em Ciência e Tecnologia, v. 9, n. 2, p. 121-147, 2016.

PAVÓN, Zenahir Siso; FERNÁNDEZ, Luigi Cuéllar. Relaciones entre las concepciones de naturaleza de la ciencia y la tecnología, y de la enseñanza y el aprendizaje de las ciencias de profesores de química en ejercicio. Una primera aproximación al esquema conceptual del profesor. Tecné, Episteme y Didaxis: TED, n. 41, p. 17-36, 2017. 
PAVÓN, Zenahir; SOTO, Iván Sánchez; FERNÁNDEZ, Luigi Cuéllar. Una experiencia de aprendizaje en formación continua de profesores de química fundamentada en naturaleza de la ciencia y tecnología. Góndola, Enseñanza y Aprendizaje de las ciencias (Bogotá, Colombia), v. 14, n. 2, p. 229-252, 2019.

QUEVEDO, Vanessa Ortega; PUENTE, Cristina Gil. La Naturaleza de la Ciencia y la Tecnología. Una experiencia para desarrollar el Pensamiento Crítico. Revista científica, v. 2, n. 35, p. 167182, 2019.

REHMAT, Abeera; BAILEY, Janelle. Technology integration in a science classroom: Preservice teachers' perceptions. Journal of Science Education and Technology, v. 23, n. 6, p. 744-755, 2014.

RESTREPO, María Mercedes Callejas; PARADA, Edgar Alberto Mendoza; PORRAS, Yair. Unidades didácticas para aprender sobre la naturaleza de la Ciencia y la Tecnología en Educación Básica (proyecto eancyt). Revista de Ensino de Ciências e Matemática, v. 3, n. 3, p. 116-128, 2012.

RIBEIRO, Thiago Vasconcelos; COLHERINHAS, Guilherme; GENOVESE, Luiz Gonzaga Roversi. O estudo de temas tecnológicos na Educação CTSA: uma experiência de alfabetização científica e tecnológica no Ensino Médio. Revista de Ensino de Ciências e Matemática, v. 7, n. 1, p. 38-58, 12 fev. 2016.

RICARDO, Elio Carlos. Concepções de tecnologia na formação inicial de professores de Física. Investigações em Ensino de Ciências, v. 25, n. 3, 2020.

RODRÍGUEZ, Andrei Steveen Moreno; DEL PINO, José Claudio. Abordagem Ciência, Tecnologia e Sociedade (CTS): perspectivas teóricas sobre educação científica e desenvolvimento na América Latina. \# Tear: Revista de Educação, Ciência e Tecnologia, v. 6, n. 2, 2017.

SANTILLI, Haydée. Science and Technology, Autonomous and More Interdependent Every Time. Science \& Education, v. 21, n. 6, p. 797-811, 2012.

SANTOS, João Paulo Silva; TENÓRIO, Alexandro Cardoso; SUNDHEIMER, Michael Lee. Visões de ciência e tecnologia entre licenciandos em física quando utilizam a robótica educacional: um estudo de caso. Investigações em Ensino de Ciências, v. 23, n. 1, p. 32-55, 2018.

SEPINI, Ricardo Pereira; ALONSO, Ángel Vázquez; MACIEL, Maria Delourdes. Mudanças de concepções atitudinais sobre a natureza da ciência e tecnologia em estudantes da escola básica após intervenção didática. Amazônia: Revista de Educação em Ciências e Matemáticas, v. 10, n. 20, p. 101-111, 2014.

SEPINI, Ricardo Pereira; MACIEL, Maria Delourdes. Como o ensino de questões relacionadas com natureza da ciência e tecnologia pode contribuir para a formação de futuros professores. Indagatio Didactica, v. 8, n. 1, p. 739-751, 2016.

SILVA, André Coelho. Uma Possível Associação entre Ciência e Tecnologia no Ensino de Ciências: o exemplo do telefone. Revista Brasileira de Ensino de Ciência e Tecnologia, v. 6, n. 3, 2013. 
STRIEDER, Roseline Beatriz. Abordagens CTS na Educação Científica no Brasil: sentidos e perspectivas. 2012. 283f. Tese (Doutorado em Ensino de Ciências). - Universidade de São Paulo, São Paulo, 2012.

STRIEDER, Roseline Beatriz; TORIJA, Beatriz Bravo; QUILEZ, Maria José Gil. Ciencia-tecnologíasociedad: ¿ Qué estamos haciendo en el ámbito de la investigación en educación en ciencias? Enseñanza de las ciencias: revista de investigación y experiencias didácticas, v. 35, n. 3, p. 29-49, 2017.

VENESSA, Devita Marlina et al. Exploring View of Nature of Science and Technology PreService Chemistry Teachers. Journal of Science Learning, v. 3, n. 1, p. 19-28, 2019.

WAIGHT, Noemi. Technology knowledge: high school science teachers'conceptions of the nature of technology. International Journal of Science and Mathematics Education, v. 12, n. 5 , p. 1143-1168, 2013. 